\title{
Commentary: TAVR-in-TAVR-Be a good host for your guest
}

\author{
Siamak Mohammadi, MD, FRCSC, and Dimitri Kalavrouziotis, MD, FRCSC
}

\author{
From the Department of Cardiac Surgery, Quebec Heart and Lung Institute, Quebec City, Quebec, Canada. \\ Disclosures: Authors have nothing to disclose with regard to commercial support. \\ Received for publication Oct 1, 2019; revisions received Oct 1, 2019; accepted for publication Oct 2, 2019; \\ available ahead of print Oct 16, 2019. \\ Address for reprints: Siamak Mohammadi, MD, FRCSC, Department of Cardiac Surgery, Quebec Heart and Lung \\ Institute, 2725 Chemin Sainte-Foy, Quebec City, Quebec G1V 4G5, Canada (E-mail: siamak.mohammadi@ \\ fmed.ulaval.ca). \\ J Thorac Cardiovasc Surg 2021;161:579-80 \\ $0022-5223 / \$ 36.00$ \\ Copyright (c) 2019 by The American Association for Thoracic Surgery \\ https://doi.org/10.1016/j.jtcvs.2019.10.019
}

Transcatheter aortic valve replacement (TAVR) prostheses' durability is a very legitimate issue, and it is increasingly being studied in a prospective fashion. ${ }^{1,2}$ It is clear that durability of TAVR prostheses is less of a concern in elderly patients, patients who are not operative candidates, and high-risk patients because of the shorter life expectancy among these patients. Given the expansion of TAVR indications to intermediate- and low-risk patients with longer life expectancy, the estimated number of potential candidates with degenerated TAVR prostheses is anticipated to increase in the future. Histologic analyses ${ }^{3}$ have shown that there is a time-dependent TAVR prosthesis degeneration, including thrombus formation, fibrosis, and calcification. These changes seem to be related to nonphysiologic hemodynamic characteristics downstream from the TAVR prosthesis, including abnormal sinus flow, turbulent stress, and restricted leaflet motion. ${ }^{4}$ Given this scenario, the questions that then arise are as follows: Can a TAVR-in-TAVR procedure be performed with good early and late results? Do the abnormal hemodynamic characteristics become accentuated with a TAVR-in-TAVR procedure? In our center and in many others, a TAVR-in-TAVR approach was traditionally performed as a bailout during in the early TAVR experience as a response to intraprocedural problems. The efficacy of TAVR-in-TAVR has not been extensively investigated, however, and it thus cannot currently be recommended as a conventional approach to replace a degenerated TAVR prosthesis in patients who are operative candidates.

In this issue of the Journal, Hatoum and colleagues ${ }^{5}$ report the elegant ex vivo study that they performed to assess the hemodynamic properties of 6 different TAVRin-TAVR combinations in an attempt to evaluate the best hemodynamic performance among the various valve types and sizes. The selected TAVR-in-TAVR combinations examined were as follows: the 26-mm older-generation Medtronic Evolut (Medtronic, Minneapolis, Minn), and the

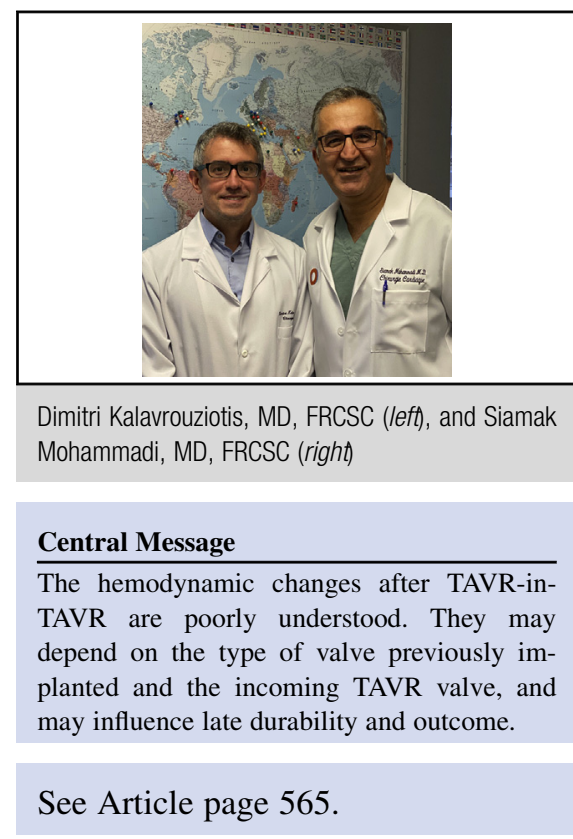

23-mm Edwards Sapien 3 (Edwards Lifesciences Corporation, Irvine, Calif) as the "host" prostheses and the 23-mm and 26-mm Evolut and the 23-mm Sapien 3 as the "guest" prostheses. The hemodynamic performance of these combinations was meticulously assessed with a flow-loop heart pulse simulator under physiologic conditions. The parameters that were examined were as follows: mean transvalvular pressure gradient; Gorlin equation-derived effective orifice area; regurgitant fraction; pinwheeling index, as a marker of localized leaflet tissue bending and poor leaflet resilience; particle image velocimetry-visualized flow characteristics through the valve; and, finally Reynolds shear stress, which correlates with increased flow turbulence and localized platelet activation.

The major conclusion of the article of Hatoum and colleagues is that, ${ }^{5}$ perhaps not surprisingly, there is enhanced hemodynamic disturbance and increased flow turbulence in TAVR-in-TAVR relative to single-prosthesis TAVR. Specifically, the findings can be summarized as follows. First, the Evolut prosthesis as host valve was associated with the highest effective orifice area and lowest flow velocities. Hatoum and colleagues ${ }^{5}$ postulate that the low radial force and flexible nitinol frame of the Evolut valve, in combination with the high radial force of the Sapien 3 guest valve, allowed for additional expansion. Second, regurgitant 
fraction was lower with the Sapien valve as host. Third, the pinwheeling index (a potential surrogate for accelerated leaflet failure) was high with the Sapien valve as host, and increased further with the Sapien-in-Sapien configuration. Hatoum and colleagues ${ }^{5}$ suggest that this was due to the rigid frame of the Sapien host valve and the intra-annular deployment of the Sapien guest valve, which may have compromised leaflet mobility of the all-Sapien TAVR-inTAVR (vs the supra-annular position of the Evolut valve leaflets).

Hatoum and colleagues ${ }^{5}$ attempted to investigate all potential hemodynamic causes for the most common types of structural and nonstructural TAVR prosthesis degenerative mechanisms in TAVR-in-TAVR. These include patientprosthesis mismatch (by evaluating pressure gradient, effective orifice area, and particle image velocimetry), perivalvular leak (by evaluating regurgitant fraction), restricted leaflet motion, flow turbulence, and risk of leaflet thrombosis (by evaluating pinwheeling index and Reynolds shear stress). The results of Hatoum and colleagues ${ }^{5}$ are certainly compelling in suggesting that certain TAVR-in-TAVR prosthetic combinations are associated with unfavorable hemodynamics, poor leaflet mobility, blood clotting cascade activation, and potentially quicker prosthetic valve degeneration. The major caveat here, however, is that any direct causal mechanism between these in vitro hemodynamic observations and late prosthetic failure of TAVR-in-TAVR valves remain hypothetical. Moreover, the exclusion of larger-sized prostheses and the inclusion of some clinically inapplicable TAVR-in-TAVR combinations may have contaminated the results, as does the absence of an actually degenerated host prosthesis with its attendant leaflet calcification or fibrosis, such as would be the case in the real world. For the practicing clinician, the major value of the study by Hatoum and colleagues ${ }^{5}$ is that it highlights the fact that patient selection for a TAVR-in-TAVR procedure should be based not only on the "host" valve size but also on the type of the host valve, which in turn may modulate the potential risk of late failure. These data may help the clinician to tailor the approach to TAVR-in-TAVR beyond just annular size considerations.

Will these hemodynamic findings be reproduced in larger TAVR prosthesis combinations? Will they be similar in TAVR--in-TAVR-in-native valve? Is the "Russian dolls" approach a safe and effective option, or is a surgical aortic valve replacement (which includes removal of native valve tissue and the failed TAVR prosthesis) preferable in the case of a late failure of a first TAVR? Is a TAVR-in-TAVR even indicated at all for patients who are operative candidates? These questions remain unanswered. Another inevitable point this article raises is the need for future studies on the impact of long-term anticoagulation among patients undergoing TAVR-in-TAVR.

Hatoum and colleagues ${ }^{5}$ are to be congratulated on their efforts to shed some light on a clinical entity that will only continue to be more prevalent in the setting of expanding TAVR indications. Their study highlights the urgent need for further research in the in vivo and clinical settings.

\section{References}

1. Blackman DJ, Saraf S, MacCarthy PA, Myat A, Anderson SG, Malkin CJ, et al. Long-term durability of transcatheter aortic valve prostheses. J Am Coll Cardiol. 2019;73:537-45.

2. Thyregod HGH, Ihlemann N, Jørgensen TH, Nissen H, Kjeldsen BJ, Petursson P, et al. Five-year clinical and echocardiographic outcomes from the NOTION randomized clinical trial in patients at lower surgical risk. Circulation. 2019;139: 2714-23.

3. Sellers SL, Turner CT, Sathananthan J, Cartlidge TRG, Sin F, Bouchareb R, et al. Transcatheter aortic heart valves: histological analysis providing insight to leaflet thickening and structural valve degeneration. JACC Cardiovasc Imaging. 2019; $12: 135-45$.

4. Hatoum H, Yousefi A, Lilly S, Maureira P, Crestanello J, Dasi LP. An in vitro evaluation of turbulence after transcatheter aortic valve implantation. J Thorac Cardiovasc Surg. 2018;156:1837-48.

5. Hatoum H, Lilly S, Maureira P, Crestanello J, Dasi LP. The hemodynamics of transcatheter aortic valve in transcatheter aortic valves. J Thorac Cardiovasc Surg. 2021;161:565-76.e2. 